\title{
Метод сегментации изображения для подсчета процентного содержания минералов с помощью авторской программы
}

\author{
Чернявский А.В., Степенщиков Д.Г. \\ Геологический институт КНЦ РАН, Anamumbl, chernyavsky@geoksc.apatity.ru; stepen@geoksc.apatity.ru
}

\begin{abstract}
Аннотация. В статье рассматривается авторская программа для определения процентного содержания минерального состава руд и продуктов технологической переработки в измельченном состоянии как в аншлифах, так и в искусственных препаратах. В основе определения лежит метод сегментации изображения с одновременным определением нескольких пороговых значений по гистограмме и интерактивной оценке их процентного содержания. Для работы программы могут быть использованы как изображения, полученные с помощью оптических методов, для минералов с высоким коэффициентом отражения, так и высококонтрастные снимки, полученные методами электронной микроскопии для минералов с низким коэффициентом отражения.

Ключевые слова: минерал, аншлиф, сегментация, гистограмма, программа, Lazarus.
\end{abstract}

\section{Image segmentation method for counting the percentage of the minerals by author's program}

\author{
Chernyavsky A.V., Stepenshchikov D.G. \\ GIKSCRAS, Apatity,chernyavsky@geoksc.apatity.ru; stepen@geoksc.apatity.ru
}

\begin{abstract}
The article considers an author's program for determining the percentage of the mineral composition of ores and products of technological processing in the ground state both in polished sections and artificial preparations. The definition is based on the method of image segmentation with simultaneous determination of several threshold values from the histogram and interactive evaluation of their percentage content. The program can use both images obtained by optical methods for minerals with a high reflection coefficient, and high-contrast images obtained by electron microscopy for minerals with a low reflection coefficient.
\end{abstract}

Key words: mineral, polished section, segmentation, histogram, program, Lazarus.

\section{Введение}

Определение относительного содержания минералов в руде или породе необходимо для характеристики ее химического состава. Использование минералогического метода оценки химического состава занимает значительно меньше времени, чем химического и не требует сложных лабораторных химико-аналитических комплексов.

При визуальном определении количества рудного вещества в образце используется метод стандартного препарата С.А. Вахромеева (рис. 1 a), разработанный только для мономинеральных руд (хромитовых, лопаритовых, сульфидных). Для точного измерения количества минералов, находящихся в срастании друг с другом, существуют специальные приставки к рудным микроскопам. Простейшими из них являются ручное устройство интеграционный столик Андина (ИСА, рис. 1 б) и полуавтоматические минералогические интеграционные устройства МИУ-1, МИУ-3. Количественный анализ минералов выполняется линейным методом (Кочурова, 1977). Современные приборы подобного назначения оборудованы автоматическими системами сканирования и расчета количества и ряда других параметров (структуры, срастаний, размера зерен и др.).

В основе программных методов определения количества рудного вещества в образце лежит метод сегментации изображения (СИ), то есть разделение изображения на области, для которых выполняется определенный критерий однородности, например выделение областей приблизительно одинаковой яркости. Цель СИ заключается в упрощении и/или изменении представления изображения, чтобы его было проще и легче анализировать. СИ обычно используется для того, чтобы выделить объекты и границы на изображениях.

Для СИ было разработано несколько методов: методы, основанные на кластеризации; методы с использованием гистограммы; метод выделения краёв; методы разрастания областей; мето- 

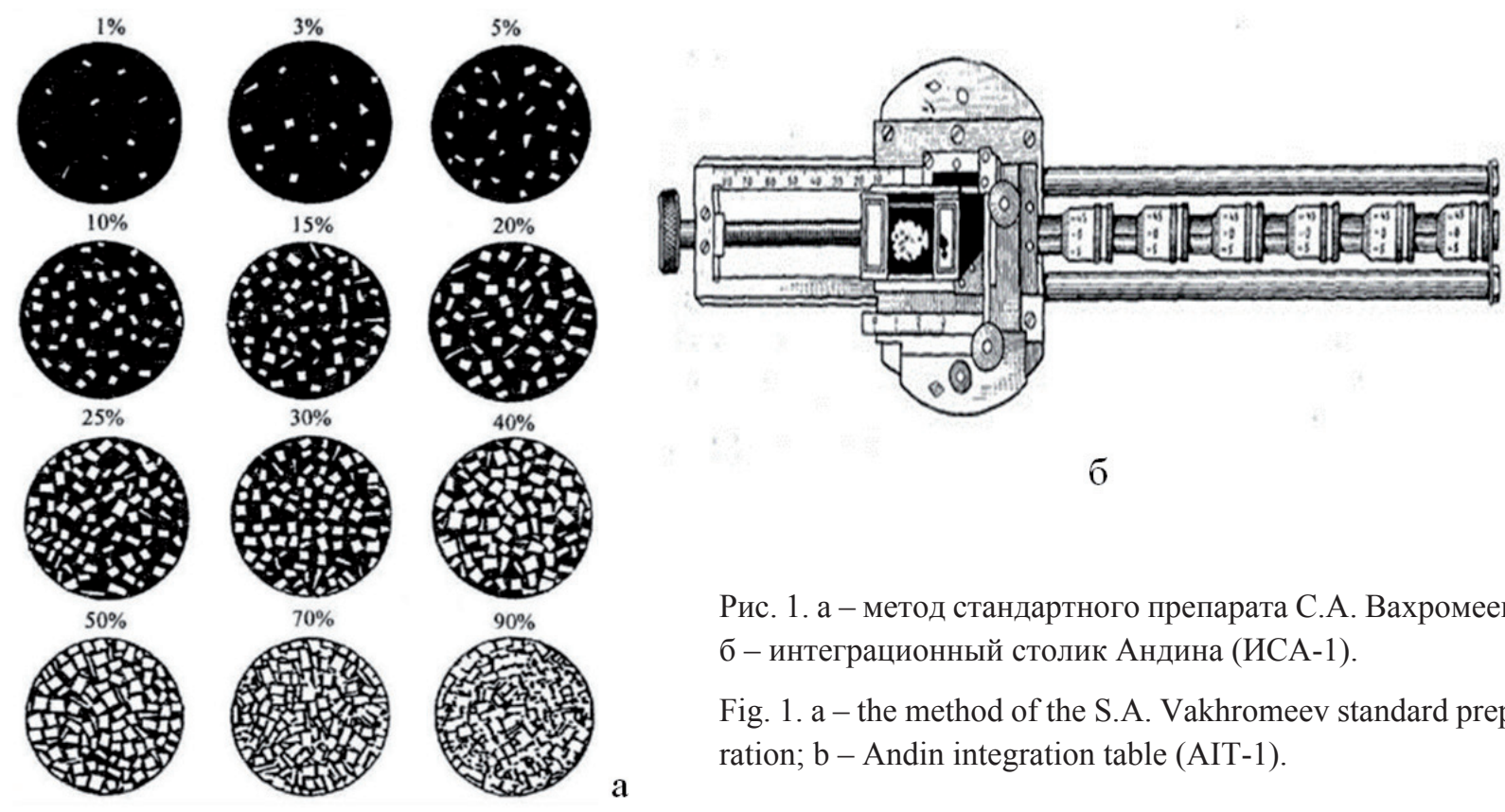

б

Рис. 1. а - метод стандартного препарата С.А. Вахромеева; б - интеграционный столик Андина (ИСА-1).

Fig. 1. a - the method of the S.A. Vakhromeev standard preparation; $\mathrm{b}$ - Andin integration table (AIT-1).

ды разреза графа; сегментация методом водораздела; сегментация с помощью модели; многомасштабная сегментация; одномерная иерархическая сегментация сигналов (Гонсалес Р., Вудс Р., 2005 ; Bradski G., Kaehler A., 2008; Comaniciu D., Meer P., 1999, 2002; Comaniciu D., Ramesh V., Meer P., 2000; Rother C., Kolmogorov V., Blake A., 2004).

Несмотря на обилие методов СИ, их применение не всегда возможно. Если речь заходит о работе с панорамными изображениями препаратов и сегментации более пяти областей, задача становиться весьма ресурсоемкой в плане компьютерных мощностей. А главное - невозможно контролировать параметры сегментации, если нет навыков владения языком программирования. К сожалению, практически все разнообразие методов не имеет конечной программной реализации. На рынке существует лишь несколько программных пакетов в которых реализованы функции сегментации. Платные: $\underline{\text { Siams, }}$ Тиксомет, ВидеоТест. Бесплатные: JMicroVision, ImageJ, FIJ, ToupView и др. Как правило, все эти программные продукты предназначены для фиксации изображения и проведения различных измерений. А вот СИ для определения количественного содержания минералов у них является слабым местом. Во всех продуктах используется метод порога гистограммы, в результате чего получается бинарное изображения только для одной области (минерала). Если же минералов несколько, то получается несколько бинарных изображений.

Из имеющихся программ наиболее продвинутой и удобной для работы является JMicroVision. В ней можно загружать панорамные изображения, на основе порога гистограммы выделять по одной области и добавлять их на общее изображение в виде цветного контура (метод выделения краев). Но программа не запоминает уже отмеченные области, то есть можно выделить и принять в расчет одну и ту же область несколько раз.

В связи со выше сказанным, авторами было разработано собственная программа для СИ, обеспечивающая желаемый функционал.

\section{Описание авторской программы}

Программа реализована в открытой среде разработки программного обеспечения на языке Object Pascal для компилятора Free Pascal (FPC) Lazarus версии 2.0.6. Выбор данной IDE обусловлен ее бесплатным распространением и простотой создания удобного интерфейса для конечного пользователя.

При запуске программы открывается основное окно, где пользователю, в частности, предлагается стандартный интерфейс работы с файлами (открытие, сохранение, закрытие файла). Программа поддерживает работу с графическими файлами формата BMP или JPEG. Изображение аншлифа из 
выбранного файла появляется в новом окне, которое можно перемещать и масштабировать независимо от основного окна; само изображение внутри окна так же может быть масштабировано. Все это сделано для удобства работы с большими изображениями. Искусственное ограничение на размер загружаемых изображений - 10000 пикселей по любой из сторон, но оно было введено только для того, чтобы большие файлы не замедляли работу программы и в принципе может быть снято.

Исходное изображение перед выводом автоматически переводится в черно-белый формат посредством усреднения значений его RGB каналов, что соответствует формату изображений, полученных методами электронной микроскопии. Одновременно в левой части основного окна выводится гистограмма с содержанием пикселей всех оттенков серого (от 0 до 255). В типичном случае, когда на изображении аншлифа присутствуют отдельные зерна минералов, то есть обширные связные однотонные области, гистограмма будет иметь мультимодальный характер.

В правой части основного окна отображается формируемая пользователем легенда - список уникальных названий областей изображения (как правило, названия минералов) и соответствующий им отличительный цвет. Список формируется независимо от загрузки изображения аншлифа и сохраняется при работе с новым файлом, что удобно в случае, когда пользователь работает с несколькими изображениями аншлифов однотипных образцов.

Привязка легенды к изображению может проходить двумя способами. При первом способе пользователь сначала выбирает элемент легенды, а затем кликает правой клавишей мыши на некоторую область изображения. После этого всем пикселям с оттенком серого в диапазоне $[x-3 ; x+3]$ присваивается цвет выбранного элемента легенды, где $x$ - оттенок пикселя, на который попал курсор мыши, а напротив выбранного элемента легенды отображается относительная площадь закрашенного участка (в \%). Одновременно на гистограмме соответствующие ее области также окраши-

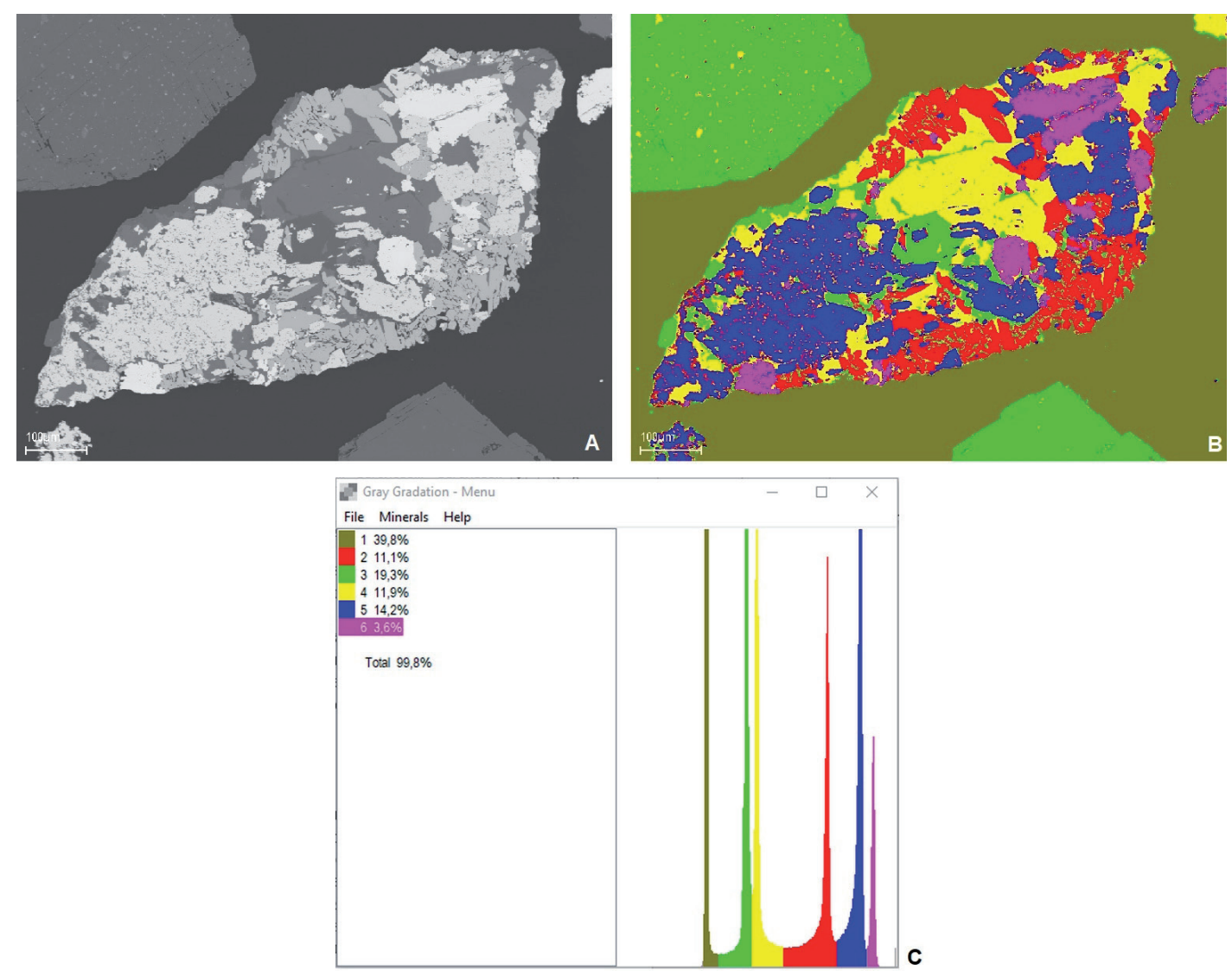

Рис. 2. Пример работы программы. А - исходное черно-белое изображение аншлифа, В - цветное изображение аншлифа, C - легенда с указанием процентного содержания цветных областей и гистограмма.

Fig. 2. An example of program work. A - original black-white image of polished section, $\mathrm{B}$ - the colored one, $\mathrm{C}$ - the legend with the percentage of the colored areas and histogram. 
ваются в соответствующий цвет. Закрашивать ранее закрашенные области нельзя. Клик правой клавишей мыши снимает цвет с выбранной области, при условии, что ее цвет совпадает с цветом выбранного элемента из списка. Второй способ аналогичен первому с той лишь разницей, что клики мыши производятся не на изображении, а на гистограмме.

Указанный способ является грубым, так как может пропускать некоторые элементы выбранной области, соответствующей минералу, но не попавшие в диапазон $[x-3 ; x+3]$. Для устранения этого на гистограмме можно корректировать границы закрашенной области либо кликом мыши, либо сочетанием клавиш.

Таким образом все области изображения (и гистограммы), соответствующие минералам, раскрашиваются в хорошо различимые цвета. Одновременно расчитывается процентное содержание минералов в аншлифе. Пример работы программы показан на рисунке 2.

В ранних версиях программы скорость обработки изображений была невысока. Поэтому стоит отметить, что в последней ее версии был использован сторонний свободно распространяемый пакет обработки изображений LazRGBGraphics 1.0 (автор - Том Грегорович), обеспечивающий прямой доступ к пикселям изображения. В результате скорость работы программы возросла в разы.

\section{Апробация авторской программы}

Оценка эффективности работы авторской программы проводилась при работе с высокоотражающими минералами (сравнение с классическими оптическими методами оценки и программой JMicroVision) и с низкоотражающими минералами (обработка результатов электронной микроскопии).

В ходе договорных работ на изготовление аншлифов и последующего их анализа, была применена методика количественного анализа минералов и их агрегатного состояния, основанная на методе СИ. Для этого была подготовлена фотодокументация полированных препаратов из продуктов Ковдорского ГОКа и созданы их панорамы. Фотодокументация проводилась со строгим контролем параметров (баланса белого и экспозиции) с использованием эталонного минерала. Площадь покрытия препарата панорамой определялась по количеству ярких минералов ( 3000 зерен), попадающих в эту панораму.

Количественный анализ проводился с применением программных и оптических методов: анализ изображения осуществлялся с помощью авторской программы и программы JMicroVision. B качестве оптического метода был использован линейный метод с применением интеграционного столика Андина.

Сравнение результатов, полученных программными и оптическими методами, показало идентичные результаты. При этом подход к решению поставленных задач с помощью программных методов оказался гораздо оперативнее и практичнее поскольку выполнение подсчета можно прервать в любой момент и при необходимости вернуться к нему в любое удобное время, без потери результатов.

Тем не менее авторская программа на текущем этапе реализации имеет недоработки. В отличие от JMicroVision она позволяет выделять и фиксировать пиковые области на гистограмме для нескольких минералов, но не имеет опции ручной коррекции площадей. В перспективе авторами разрабатывается возможность ручной коррекции, а также количественной оценки раскрывшихся минеральных видов и сростков.

Для рудных минералов с низким коэффициентом отражения традиционные оптические методы бессильны: различимость минеральных видов в искусственных препаратах и аншлифах при работе в отраженном свете стремится к нулю - изображение представляет собой «серую массу» с едва заметными границами зерен. В этом случае на помощь приходит электронная микроскопия с возможностью получения высококонтрастных изображений и оценкой химического состава минералов. Но и здесь невозможно получить оценку процентного содержания минеральных видов имеющимися сторонними программами.

В ходе полевых работ в 2018 году были отобраны две технологические пробы анкилит- и бастнезит-содержащих руд из канав участка Неске-Вара. Пробы были разделены на 11 фракций, каждая из которых была сфотографирована. Часть каждой фракции была отобрана и использована 
для создания искусственных шлифов. Исследование фазовой и внутрифазовой неоднородности индивидов, а также изучение химического состава минералов осуществлялось методами оптической и электронной микроскопии на приборах, имеющихся в распоряжении ГИ КНЦ РАН: оптическом микроскопе Axioplan с регистрацией минеральных объектов с помощью видеокамеры и сканирующем электронном микроскопе (CЭM) LEO-1450 с энергодисперсионной приставкой Quantax (аналитик А. Тележкин).

Для подсчета процентного содержания минеральных видов с помощью авторской программы было сделано 10-15 снимков для каждой стадии дробления породы. Съемка выполнялась на СЭМ LEO-1450 при одинаковых условиях $(\mathrm{Br}=50.4 \%$; Cont $=35 \%$; Iprobe=1.1 nA; $20 \mathrm{kV})$, подобранных так, чтобы добиться максимально контрастного разделения минеральных видов.

В результате работы можно сделать вывод, что авторская программа является весьма эффективным инструментом для подсчета процентного содержания минеральных видов, зафиксированных с помощью оптических методов и методов электронной микроскопии.

Работа выполнена в рамках тем НИР ГИ КНЦ РАН № 0226-2019-0052 и НИР ГИ КНЦ РАН № 0226-2019-0053, AAAA-A19-119100290147-7.

\section{Литература}

1. $\quad$ Гонсалес Р., Вудс Р. Цифровая обработка изображений. М. Изд-во: Техносфера. 2005. 1072 с.

2. Кочурова Р.Н. Основы практической петрографии. Л. Изд-во: Ленинградского университета. 1977. 176 c.

3. Bradski G., Kaehler A. Learning OpenCV. O’Reilly Media, Inc. 2008. 556 p.

4. Comaniciu D., Meer P. Mean shift analysis and applications // Proceedings of the Seventh IEEE International Conference on Computer Vision. 1999. 2. P. 1197-1203. https://www.doi.org/10.1109/ICCV.1999.790416.

5. Comaniciu D., Ramesh V., Meer P. Real-Time Tracking of Non-Rigid Objects Using Mean Shift // Proceedings IEEE Conference on Computer Vision and Pattern Recognition. 2000. V. 2. P. 142-149. https://www.doi. org/10.1109/CVPR.2000.854761.

6. Comaniciu D., Meer P. Mean Shift: A Robust Approach Toward Feature Space Analysis // IEEE Transactions on Pattern Analysis and Machine Intelligence. 2002. V. 24(5) P. 603-619. https://www.doi. org/10.1109/34.1000236.

7. Rother C., Kolmogorov V., Blake A. Grabcut - interactive foreground extraction using iterated graph cuts // ACM SIGGRAPH 2004 Papers. 2004. P. 309-314. https://www.doi.org/10.1145/1186562.1015720. 